\title{
A SURVEY ON CLASSIFICATION OF LIVER TUMOUR FROM ABDOMINAL COMPUTED TOMOGRAPHY USING MACHINE LEARNING TECHNIQUES
}

\author{
Gunasundari $\mathbf{S}^{\mathbf{1}}$ \\ ${ }^{1}$ Associate Prof, Velammal Engineering College, Chennai \\ Swetha $\mathbf{R}^{2}$ \\ ${ }^{2}$ Student, Velammal Engineering College, Chennai
}

Article DOI: https://doi.org/10.36713/epra6570

\begin{abstract}
Pattern recognition is a significant area of research in medicine because many applications like diagnostic system benefit from it. The aim of this research is to analyze developments of liver cancer detection using machine learning techniques for liver disease. The study highlights how liver cancer diagnosis is assisted using machine learning with supervised, unsupervised and deep learning techniques. Several state of art techniques are compared based on performance measures such as accuracy, sensitivity, specificity. Finally, challenges are also highlighted for possible future work.
\end{abstract}

KEYWORDS: Machine Learning, Liver, Liver disease, Computer Aided Diagnosis system, Liver Cancer, Computed Tomography

\section{INTRODUCTION}

Liver disease is any trouble of liver function that causes sickness. There are many different types of liver disease. The benign tumors are not cancerous, and doctors can remove them. The benign tumors cells do not spread to tissues around them or to other parts of the body. There are several types of benign liver tumors; they are hemangioma, hepatic adenoma, cysts, lipoma, fibroma, leiomyoma, and focal nodular hyperplasia (FNH). The most common type of benign tumor is hemangioma. This type of benign tumor consists of masses of twisted congested blood vessels and they start in blood vessels. The second most common tumor of liver is FNH. This tumor is the result of congenital arteriovenous malformation hepatocyte response. Although FNH tumors are benign, it is very difficult to differentiate from liver cancer and doctors remove the tumor sometimes when the diagnosis is unclear.
Primary liver cancer is the sixth most frequent cancer globally. Liver cancer is the second leading cause of cancer death. Cancer cells can invade and damage nearby tissues and organs. Most frequent primary liver cancers begin in hepatocytes (liver cells). This type of cancer is called hepatocellular carcinoma (HCC) or hepatoma. Hepatocellular Carcinoma (HCC) is the most common kind of liver cancer, which causes up to $80 \%$ of victims (Stewart et al 2014) Some of the other malignant tumors include cholangiocarcinoma, sarcoma, and hepatoblastoma. According to GLOBOCAN report (Bray et al 2018) that liver cancer is the 6th and 7th causes of demises in men and females respectively

Advances in medical imaging and image processing techniques have greatly enhanced the interpretation of medical images. Radiographic imaging modalities like Computed Tomography (CT) and Magnetic Resonance Imaging (MRI) help in liver 
cancer diagnosis. Medical imaging based on CT is suitable for the detection of liver, kidney, and lung cancer diseases. In fact, a large number of Computer Aided Diagnostic System (CAD) systems have been employed for assisting physicians in the early detection of liver, kidney, breast, and lung cancers.

\section{LITERATURE SURVEY}

Different types of liver cancer classification using machine assistance have opened up a new research area for early detection of cancer. Many authors have surveyed techniques for liver disease diagnosis. (Fujita et al(2010), Rathore et al(2011), Gunasundari et al(2013), Anwar et al(2018), Brunetti et al (2019), Saba (2020), Nayantara et al (2020)). Chen et al (1998) developed system based on fractal geometry and modified probabilistic neural network for the CT liver image classification. Gletsos et al (2001) proposed a computer-aided diagnostic system for the classification of hepatic lesions from CT images. The use of texture features, the application of dimensionality reduction with sequential forward floating selection (SFFS), and the choice of a classifier consisting of three sequentially placed neural networks, have resulted in a total classification performance of $98 \%$, for four classes of hepatic tissue.

Mougiakakou et al (2003) proposed a CAD system based on texture features and a multiple classification scheme for the characterization of four types of hepatic tissue from CT images has been presented. In Gletsos et al (2003), the classifier module consists of three sequentially placed feed-forward neural networks (NNs). The first NN classifies into normal or pathological liver regions. The pathological liver regions are characterized by the second $\mathrm{NN}$ as cyst or "other disease." The third NN classifies "other disease" into hemangioma or hepatocellular carcinoma. Three feature selection techniques have been applied to each individual $\mathrm{NN}$ : the sequential forward selection, the sequential floating forward selection, and a genetic algorithm for feature selection. Result shows that genetic algorithm yields lower dimension feature vectors and improved classification performance.

In Valavanis et al (2004), an ensemble of classifiers has been constructed by using neural network to discriminate four hepatic tissue types: normal liver, hepatic cyst, hemangioma and hepatocellular carcinoma from non-enhanced CT images. Mala et al (2005) extracted Wavelet based texture features and used it to train the Probability Neural Network (PNN) to classify the liver as fatty liver or cirrhotic liver. Mala et al $(2006,2008)$ extracted Biorthogonal wavelet based texture features and used it to train the PNN to classify the liver tumor as hepatocellular carcinoma, cholangiocarcinoma, hepatocellular adenoma and hemangioma

A classifier based on the support vector machine (SVM) is proposed for automatic classification in liver disease (Huang et al (2006), Lee et al (2007 a)).
Lee et al (2007 b) introduced a kernel-based classifier for liver disease distinction of computer tomography (CT) images. In Stavroula et al (2007), for each CT liver ROI, five types of texture feature sets, based on first order statistics, spatial gray level dependence matrices, gray level difference matrices, Laws' texture energy measures, and fractal dimension measurements, are extracted resulting in a total of 89 features. A Genetic Algorithm based feature selection method was applied to feature sets, when dimensionality reduction was desired, while two alternative Ensemble of Classifiers were investigated consisting solely of NN classifiers, or of a combination of NN and statistical classifiers. A plurality and a weighted voting scheme are used to combine the outputs of the primary classifiers of the examined CAD systems

Bharathi et al (2008) observed that the Zernike moment features are to be efficient in distinguishing the soft liver tissues into normal and abnormal. In Wang et al. 2009, an experienced radiologist identified ROI with CT liver images. Texture features based on First Order Statistics (FOS), SGLDM, Gray Level Run Length Method (GLRLM), and Gray Level Difference Method (GLDM) are extracted for each ROI. Multi class SVM is used to classify liver diseases into primary hepatic carcinoma, hemangioma, and normal liver.

Kumar et al (2010) proposed a novel feature extraction scheme based on multiresolution fast discrete curvelet transform (FDCT) and employed ANN classifier using FDCT features for computer-aided diagnosis of liver disease. Kondo et al (2011) proposed the hybrid Group Method of Data Handling type neural network algorithm using the artificial intelligence for the medical image diagnosis of liver cancer. Stoean et al. 2011 developed automatic tool to differentiate five degrees of liver fibrosis. Hill climbing algorithm is used for selecting best features. Cooperative coevolutionary algorithm is used to evolve set of rules that is based on training samples and then rules are used for characterizing between different degrees of fibrosis for the test case.

Kumar et al (2012) concluded that contourlet based features performed better than the Gray Level textural features. A tandem feature selection mechanism and evolutionary trained neural network model is proposed for the classification of stages for liver fibrosis within chronic hepatitis C (Gorunescu, 2012). Kumar et al. (2013) proposed liver CAD system to classify HCC and hemangioma. Wavelet coefficients and Contourlet coefficients are reduced using Principal Component Analysis (PCA). The reduced features are fed into PNN for classification of benign tumor from malignant and the accuracy got using CCCM feature is 96.7, which is higher than other features

In Zhou 2014, data visualization and classification method is presented to distinguish between healthy and diseased livers for early-stage diagnosis of liver 


\section{EPRA International Journal of Research and Development (IJRD)}

disease. Glowworm swarm optimization is used for optimizing parameters width and penalty constant of Support Vector Data Description that is used to improve diagnostic accuracy. Gunasundari et al (2016) applied Velocity bounded Boolean PSO for feature selection problem to select elite features from liver cancer data. When applied in liver CAD system, proposed algorithm selects elite features, which is used to classify hepatoma and cholangiocarcinoma as malignant and hemangioma and FNH as benign.

Alahmer et al (2016) proposed CAD system which divides a segmented lesion into three areas, i.e. inside, outside and border areas. Features extracted from the three areas are used to build a new feature vector to feed a classifier for better differentiation between benign and malignant lesions. Sayed et al (2016) proposed a fully automatic CAD system for liver and lesion segmentation and for liver disease diagnosis. The liver segmentation approach is based on a hybrid approach of fuzzy clustering and grey wolf optimisation, while a fast fuzzy c-means technique is employed for lesion segmentation. Shape and texture features are extracted from lesions and employed in a classification stage using a support vector machine classifier.

Chang et al (2017) proposed a computer-aided diagnosis (CAD) system to diagnose liver cancer using the features of tumors obtained from multiphase CT image. Three kinds of features were obtained for each tumor, including texture, shape, and kinetic curve. The texture was quantified using 3dimensional (3-D) texture data of the tumor based on the grey level co-occurrence matrix (GLCM). Backward elimination was used to select the best combination of features, and binary logistic regression analysis was used to classify the tumors with leaveone-out cross validation

Kuo (2018) proposed a computer-aided diagnosis system with texture analysis for liver tumors in CT images. Improved PSO algorithm and SVM are combined for reducing features to less than 20 by the proposed two-stage selection method. Meanwhile, fuzzy logic weight value is adopted to determine the final classification result.

Gunasundari et al (2018) proposed Multiswarm Heterogeneous Binary Particle Swarm Optimization algorithm and tested in the feature selection phase of intelligent liver and kidney cancer diagnostic systems to select elite features from the liver cancer data. MHBPSO is a cooperation algorithm, which includes BPSO and its three variants such as Boolean PSO (BoPSO), Self Adjusted Hierarchical Boolean PSO (SAHBoPSO), and CatfishSelf Adjusted Hierarchical Boolean PSO (CSAHBoPSO). The elite feature sets are extracted using SAHBoPSO, CSAHBoPSO, and MHBPSO1 and they are used to classify the liver disease as benign or malignant with minimum error rate. Results show that MHBPSO1 is superior in selecting elite features.
Gunasundari et al 2019 proposed adjusted BPSO (ABPSO) to improve the performance of feature selection and the convergence speed of BPSO. The algorithm is verified in the feature selection module of liver CAD system. Yamashita et al (2020) shows the feasibility of using Convolutional Neural Network $(\mathrm{CNN})$ to assign LI-RADS categories (LR-1-5) to liver observations on multiphase CT and MRI from a relatively small sample of images. Li et al (2019) designed a liver cancer diagnosis system that is based on BP neural network after dimensionality reduction by $\mathrm{PCA}$, which combines multiple features and multi-phase information. In Al-Shabi et al (2019), the performance of SVM, MLP and GRNN has been examined in classifying the liver tissues (affected or unaffected) dataset.

Das et al (2019) proposed a new system called as watershed Gaussian based deep learning (WGDL) technique for effective delineate the cancer lesion in CT images of the liver. The segmented textural features were fed to deep neural network (DNN) classifier for automated classification of three types of liver cancer i.e. hemangioma (HEM), hepatocellular carcinoma (HCC) and metastatic carcinoma (MET). Sadeque et al (2019) presents an automated method of detecting liver cancer in abdominal CT images and classifying them using the histogram of oriented gradient - support vector machine (HOG-SVM) algorithm. In Rajathi et al (2019), to classify Chronic liver disease (CLD), the hybrid whale optimization algorithm with simulated annealing (WOA-SA) is used in selecting an optimal set of features. A hybrid ensemble classifier with support vector machine, $\mathrm{k}-$ Nearest Neighbor (k-NN), and random forest (RF) classifiers are used to classify liver diseases.

Dong et al (2020) presented the Hybridized fully convolutional neural network (HFCNN) method for liver cancer and lesion identification and segmentation. In Li et al (2020), Liver and tumor segmentation are implemented by a fully convolutional networks using a weighted loss function. Then the output of FCN is combined with the original CT image to construct a 4- channel image data as the input of a 9layers $\mathrm{CNN}$ classifier.

Shapira et al (2020) implemented a weaklysupervised convolutional neural network $(\mathrm{CNN})$ that learns liver lesion localisation without pixel-level ground truth annotations. Regions-of-interest are selected automatically based on the localisation results and are used to train a second CNN for liver lesion classification (healthy, cyst, hypodense metastasis). Lee et al (2020) designed multiple prediction models for 5-year metachronous liver metastasis (5YLM) using combinations of clinical variables (age, sex, T stage, N stage) and top principal components (PCs), with logistic regression classification

Literature review reveals [Table 1] that only some diseases like HCC and hemangioma are considered by many authors. Diseases like cholangiocarcinoma, 


\section{EPRA International Journal of Research and Development (IJRD)}

FNH, Fatty liver, cirrhosis and other diseases are not focused by many authors. Therefore, there is a requirement for a liver CAD system to characterize the benign tumor from the malignant tumor, which considers the rare diseases.

Table 1: Performance of existing liver CAD system

\begin{tabular}{|c|c|c|c|c|}
\hline $\begin{array}{l}\text { Author } \\
\text { Name } \\
\text { Year }\end{array}$ & $\begin{array}{l}\text { Textural Features and Clas- } \\
\text { sifier }\end{array}$ & Classifier & Disease & Performance \\
\hline Chen 1998 & $\begin{array}{l}\text { detect-before-extract (DBE) } \\
\text { system } \\
\text { fractal feature information } \\
\text { and the gray-level co- } \\
\text { occurrence matrix }\end{array}$ & $\begin{array}{l}\text { Modified } \\
\text { PNN }\end{array}$ & $\begin{array}{l}\text { Hepatoma and } \\
\text { Hemangioma }\end{array}$ & accuracy $=83 \%$ \\
\hline $\begin{array}{l}\text { Gletsos } \\
2001\end{array}$ & $\begin{array}{l}\text { Angular second moment, con- } \\
\text { trast, correlation, sum of } \\
\text { squares, inverse difference } \\
\text { moment, entropy, homogenei- } \\
\text { ty, cluster tendency } \\
\text { Feature selection : SFFS }\end{array}$ & $\begin{array}{c}\text { Feed- } \\
\text { Forward NN }\end{array}$ & $\begin{array}{l}\text { Normal liver, } \\
\text { hepatic cysts, } \\
\text { hemangioma, } \\
\text { and HCC }\end{array}$ & accuracy $=98 \%$ \\
\hline $\begin{array}{l}\text { Gletsos } \\
2003\end{array}$ & $\begin{array}{l}48 \text { texture characteristics } \\
\text { from spatial gray-level co- } \\
\text { occurrence matrices- Angular } \\
\text { Second Moment, Contrast, } \\
\text { correlation, sum of Square- } \\
\text { Variance, Inverse Difference } \\
\text { Moment, Entropy, Homoge- } \\
\text { neity, Cluster Tendency } \\
\text { Feature Selection: } \\
\text { SFS, SFFS, Genetic Algorithm } \\
\text { (GA) }\end{array}$ & $\begin{array}{c}\text { Feed- } \\
\text { forward NN }\end{array}$ & $\begin{array}{l}\text { Normal liver, } \\
\text { hepatic cysts, } \\
\text { hemangioma, } \\
\text { and HCC }\end{array}$ & accuracy $=97 \%$ \\
\hline $\begin{array}{l}\text { Mougiakako } \\
\text { u } 2003\end{array}$ & $\begin{array}{c}\text { FOS, SGLDM, } \\
\text { GLDM, TEM, and } \\
\text { FDM } \\
\text { Feature Selection : GA }\end{array}$ & $\begin{array}{l}\text { 4-class NN- } \\
\text { Back propa- } \\
\text { gation Algo- } \\
\text { rithm }\end{array}$ & $\begin{array}{l}\text { Normal liver, } \\
\text { hepatic cyst, } \\
\text { hemangioma, } \\
\text { and HCC. }\end{array}$ & $\begin{array}{c}\text { accuracy }= \\
97 \%\end{array}$ \\
\hline $\begin{array}{c}\text { Valavanis } \\
2004\end{array}$ & $\begin{array}{l}\text { texture features } \\
\text { Feature Selection: GA }\end{array}$ & $\begin{array}{l}\text { Two Neural } \\
\text { Network } \\
\text { (NN) and } \\
\text { three statis- } \\
\text { tical classifi- } \\
\quad \text { ers }\end{array}$ & $\begin{array}{l}\text { Normal, hepatic } \\
\text { cyst, hemangi- } \\
\text { oma, hepatocel- } \\
\text { lular carcinom }\end{array}$ & $\begin{array}{c}\text { accuracy }= \\
90.63 \%\end{array}$ \\
\hline Mala 2005 & $\begin{array}{c}\text { Orthogonal wavelet based } \\
\text { texture analysis }\end{array}$ & PNN & $\begin{array}{l}\text { Fatty or cirrho- } \\
\text { sis } \\
\end{array}$ & $\begin{array}{c}\text { accuracy }=95 \%, \\
\text { sensitivity }=96 \% \\
\text { and specificity = } \\
94 \%\end{array}$ \\
\hline Huang 2006 & $\begin{array}{c}\text { Auto-Covariance Texture Fea- } \\
\text { tures }\end{array}$ & SVM & $\begin{array}{l}\text { Malignancy and } \\
\text { hemangioma }\end{array}$ & $\begin{array}{c}\text { accuracy } 81.7 \% \text {, } \\
\text { sensitivity } 75.0 \% \\
\text { specificity } 88.1 \% \\
\text { positive predic- } \\
\text { tive value } 85.7 \% \\
\text { negative predic- } \\
\text { tive value } 78.7 \% \text {. }\end{array}$ \\
\hline
\end{tabular}


EPRA International Journal of Research and Development (IJRD)

Volume: 6 | Issue: 3 | March 2021

- Peer Reviewed Journal

\begin{tabular}{|c|c|c|c|c|}
\hline Lee $2007 \mathrm{a}$ & $\begin{array}{l}\text { gray level and co-occurrence } \\
\text { matrix features and region- } \\
\text { based shape descriptors }\end{array}$ & SVM & $\begin{array}{l}\text { cysts, hepatoma, } \\
\text { cavernous he- } \\
\text { mangioma, and } \\
\text { normal tissue }\end{array}$ & $\begin{array}{c}\text { Average AUC of } \\
\text { distinction is } 0.91\end{array}$ \\
\hline Lee 2007 b & $\begin{array}{l}\text { gray level co-occurrence ma- } \\
\text { trix, and shape descriptors } \\
\text { sequential forward selection } \\
\text { (SFS) algorithm }\end{array}$ & $\begin{array}{c}\text { kernel-based } \\
\text { classifier }\end{array}$ & $\begin{array}{l}\text { cyst, hepatoma } \\
\text { and cavernous } \\
\text { hemangioma }\end{array}$ & $\begin{array}{c}\text { Average AUC of } \\
\text { distinction is } 0.91\end{array}$ \\
\hline $\begin{array}{l}\text { Mougiakako } \\
\text { u } 2007\end{array}$ & $\begin{array}{c}\text { FOS, SGLDM, } \\
\text { GLDM, TEM, and } \\
\text { FDM } \\
\text { Feature Selection : GA }\end{array}$ & $\begin{array}{l}5 \text { Multilayer } \\
\text { Perceptron } \\
\text { NN }\end{array}$ & $\begin{array}{l}\text { Normal liver, } \\
\text { Hepatic cyst, } \\
\text { hemangioma, } \\
\text { and HCC }\end{array}$ & $\begin{array}{l}\text { accuracy } \\
=84.96 \%\end{array}$ \\
\hline $\begin{array}{l}\text { Bharathi } \\
2008\end{array}$ & $\begin{array}{c}\text { Zernike moments and Legen- } \\
\text { dre moments } \\
\text { SFS,SBS }\end{array}$ & $\begin{array}{l}\text { Nearest } \\
\text { mean classi- } \\
\text { fier } \\
\end{array}$ & $\begin{array}{c}\text { normal and } \\
\text { abnormal with } \\
\text { HCC }\end{array}$ & $\begin{array}{c}\text { Zernike feature } \\
\text { vector } \\
\text { normal } 98.33 \% \\
\text { HCC } 90.67 \% \text {. } \\
\text { Legendre fea- } \\
\text { ture vector } \\
\text { normal } 97.66 \% \\
\text { HCC } 81.67 \%\end{array}$ \\
\hline Mala 2008 & $\begin{array}{l}\text { wavelet based texture fea- } \\
\text { tures }\end{array}$ & PNN & $\begin{array}{l}\text { hepatocellular } \\
\text { carcinoma } \\
\text {,cholangio car- } \\
\text { cinoma, heman- } \\
\text { gioma and hepa- } \\
\text { toadenoma }\end{array}$ & accuracy $=90.2 \%$ \\
\hline Wang 2009 & $\begin{array}{c}\text { FOS, SGLDM, GLRLM and } \\
\text { GLDM }\end{array}$ & $\begin{array}{l}\text { Multi Class } \\
\text { SVM }\end{array}$ & $\begin{array}{l}\text { HCC, Hemangi- } \\
\text { oma, Normal }\end{array}$ & $97.78 \%$ \\
\hline $\begin{array}{c}\text { Kumar } \\
2010\end{array}$ & FDCT & $\begin{array}{l}\text { Feed For- } \\
\text { ward NN }\end{array}$ & $\begin{array}{l}\text { HCC and he- } \\
\text { mangioma }\end{array}$ & $\begin{array}{l}\text { Curvelet - 93.3\% } \\
\text { Wavelet - 88.9\% } \\
\end{array}$ \\
\hline $\begin{array}{c}\text { Stoean } \\
2011 \\
\end{array}$ & $\begin{array}{c}24 \text { indicators like Stiffness, } \\
\text { Sex, BMI, Glycemia etc. }\end{array}$ & CCEA & $\begin{array}{c}\text { Different de- } \\
\text { grees of fibrosis }\end{array}$ & $\begin{array}{l}\text { accuracy } \\
=62.11 \% \\
\end{array}$ \\
\hline $\begin{array}{c}\text { Gorunescu } \\
2012\end{array}$ & $\begin{array}{c}25 \text { indicators like Stiffness, } \\
\text { Sex, BMI, Glycemia etc. } \\
\text { GA: Feature Selector }\end{array}$ & MLP & $\begin{array}{l}\text { Different de- } \\
\text { grees of fibrosis }\end{array}$ & $\begin{array}{l}\text { accuracy } \\
=65.21 \%\end{array}$ \\
\hline $\begin{array}{c}\text { Gun- } \\
\text { asundari } \\
2012 \\
\end{array}$ & $\begin{array}{c}\text { Co-occurrence matrix and } \\
\text { Fast discrete Curvelet trans- } \\
\text { form }\end{array}$ & $\begin{array}{c}\text { BPN, PNN } \\
\text { and CFBPN. }\end{array}$ & $\begin{array}{l}\text { hemangioma, } \\
\text { and HCC }\end{array}$ & $\begin{array}{l}\text { BPN }-96 \% \\
\text { PNN - 96\% } \\
\text { CFBPN }-96 \% \\
\end{array}$ \\
\hline $\begin{array}{c}\text { Kumar } \\
2012\end{array}$ & $\begin{array}{l}\text { FOS, GLCM,CCFOS, CCCMs } \\
\text { Feature Selection : PCA }\end{array}$ & PNN & $\begin{array}{l}\text { HCC and he- } \\
\text { mangioma }\end{array}$ & $\begin{array}{c}\text { FOS }-79 \% \\
\text { GLCM }-86 \% \\
\text { CCFOS }-93 \% \\
\text { CCCM }-94 \%\end{array}$ \\
\hline $\begin{array}{c}\text { Kumar } \\
2013\end{array}$ & $\begin{array}{c}\text { Gray Level, Co-occurrence } \\
\text { features, wavelet co-efficient } \\
\text { and contourlet coefficient } \\
\text { statistics } \\
\text { PCA as feature Selector }\end{array}$ & PNN & $\begin{array}{l}\text { Benign from } \\
\text { malignant } \\
\text { HCC and he- } \\
\text { mangioma }\end{array}$ & CССМ $-96.7 \%$ \\
\hline Zhou 2014 & $\begin{array}{l}13 \text { features Age, Gender, ALT, } \\
\text { AST etc. } \\
\text { Glowworm swarm optimiza- } \\
\text { tion }\end{array}$ & $\begin{array}{l}\text { SVDD Sup- } \\
\text { port Vector } \\
\text { Data De- } \\
\text { scription }\end{array}$ & $\begin{array}{l}\text { Healthy and } \\
\text { Unhealthy }\end{array}$ & $\begin{array}{l}\text { accuracy } \\
=84.28 \%\end{array}$ \\
\hline Gun- & Textural Features & PNN, SVM & Hepatoma, & accuracy \\
\hline
\end{tabular}


EPRA International Journal of Research and Development (IJRD) Volume: 6 | Issue: 3 | March 2021 - Peer Reviewed Journal

\begin{tabular}{|c|c|c|c|c|}
\hline $\begin{array}{l}\text { asundari } \\
2016\end{array}$ & $\begin{array}{l}\text { Velocity Bounded Boolean } \\
\text { PSO }\end{array}$ & & $\begin{array}{l}\text { cholangiocarci- } \\
\text { noma, hemangi- } \\
\text { oma and FNH }\end{array}$ & $\begin{aligned} \mathrm{PNN} & =82.86 \% \\
\mathrm{SVM} & =82.86 \%\end{aligned}$ \\
\hline Sayed 2016 & $\begin{array}{l}\text { FOS, Texture (SGLDM) and } \\
\text { shape }\end{array}$ & SVM & $\begin{array}{c}\text { Benign from } \\
\text { Malignant }\end{array}$ & accuracy $=97 \%$ \\
\hline $\begin{array}{l}\text { Alahmer } \\
2016\end{array}$ & Difference of feature & SVM and NB & $\begin{array}{l}\text { cyst, hemangi- } \\
\text { oma, HCC, and } \\
\text { metastasis }\end{array}$ & accuracy $=97.5 \%$ \\
\hline Chang 2017 & $\begin{array}{l}\text { SGLDM, Shape, Kinetic curve } \\
\text { characteristic }\end{array}$ & $\begin{array}{l}\text { logistic re- } \\
\text { gression } \\
\text { analysis }\end{array}$ & $\begin{array}{l}\text { Benign from } \\
\text { malignant }\end{array}$ & $\begin{array}{l}\text { accuracy } \\
=81.69 \%\end{array}$ \\
\hline Kuo 2018 & $\begin{array}{c}\text { Statistical texture features } \\
\text { particle swarm optimization } \\
\text { (PSO) algorithm, }\end{array}$ & SVM & $\begin{array}{l}\text { Benign and Ma- } \\
\text { lignant }\end{array}$ & $\begin{array}{c}\text { Accuracy } 84.53 \% \\
\text { Sensitivity } 80 \% \\
\text { Specificity } \\
88.09 \% \\
\text { positive predic- } \\
\text { tive value (PPV) } \\
88.77 \% \\
\text { negative predic- } \\
\text { tive value (NPV) } \\
84.01 \% \\
\end{array}$ \\
\hline $\begin{array}{l}\text { Gun- } \\
\text { asundari } \\
2018\end{array}$ & $\begin{array}{c}\text { Textural Features } \\
\text { Multiswarm heterogeneous } \\
\text { binary PSO }\end{array}$ & PNN, SVM & $\begin{array}{c}\text { Hepatoma, } \\
\text { cholangiocarci- } \\
\text { noma, hemangi- } \\
\text { oma and FNH }\end{array}$ & $\begin{array}{c}\text { Accuracy } \\
\text { PNN }=82.86 \% \\
\text { SVM }=82.86 \%\end{array}$ \\
\hline $\begin{array}{l}\text { Gun- } \\
\text { asundari } \\
2019\end{array}$ & $\begin{array}{c}\text { Textural Features } \\
\text { Embedded Binary PSO }\end{array}$ & PNN, SVM & $\begin{array}{l}\text { Hepatoma, } \\
\text { cholangiocarci- } \\
\text { noma, hemangi- } \\
\text { oma and FNH }\end{array}$ & $\begin{array}{c}\text { Accuracy } \\
\text { PNN }=77.14 \% \\
\text { SVM }=82.86 \%\end{array}$ \\
\hline $\begin{array}{c}\text { Yamashita } \\
2020\end{array}$ & & Deep CNN & HCC & Accuracy $60.4 \%$ \\
\hline Li 2019 & $\begin{array}{c}\text { Statistical Features } \\
\text { PCA }\end{array}$ & $\begin{array}{l}\text { Back Propa- } \\
\text { gation neural } \\
\text { network }\end{array}$ & $\mathrm{HCC}$ & $\begin{array}{c}\text { accuracy = } \\
96.98 \%\end{array}$ \\
\hline Das 2019 & Textural Features & $\begin{array}{l}\text { deep neural } \\
\text { network } \\
\text { (DNN) classi- } \\
\text { fier } \\
\end{array}$ & $\begin{array}{l}\text { Hemangioma, } \\
\text { HCC, metastatic } \\
\text { carcinoma }\end{array}$ & $\begin{array}{c}\text { accuracy }= \\
99.38 \%\end{array}$ \\
\hline $\begin{array}{l}\text { Rajathi } \\
2019\end{array}$ & $\begin{array}{l}\text { Textural Features } \\
\text { Feature Selection: Hybrid } \\
\text { Whale Optimization with } \\
\text { Simulated Annealing }\end{array}$ & $\begin{array}{c}\text { Ensemble } \\
\text { Classifier } \\
\text { (SVM, k- } \\
\text { Nearest } \\
\text { Neighbour, } \\
\text { Random } \\
\text { Forest) }\end{array}$ & $\begin{array}{l}\text { Chronic Liver } \\
\text { Disease }\end{array}$ & accuracy $=98 \%$ \\
\hline $\begin{array}{l}\text { Sadeque } \\
2019\end{array}$ & $\begin{array}{c}\text { Histogram of oriented gradi- } \\
\text { ent }\end{array}$ & SVM & liver cancer & accuracy $=94 \%$ \\
\hline Lee 2020 & Clinical Variables & CNN & $\begin{array}{l}\text { metachronous } \\
\text { liver metastasis }\end{array}$ & AUC- 0.747 \\
\hline Dong 2020 & & $\begin{array}{l}\text { Hybridized } \\
\text { Fully Convo- } \\
\text { lutional Neu- } \\
\text { ral Network } \\
\text { (HFCNN) }\end{array}$ & $\begin{array}{l}\text { Liver Cancer } \\
\text { Detection }\end{array}$ & $\mathrm{ROC}=0.96$ \\
\hline Li 2020 & & CNN & hepatocellular & Accuracy \\
\hline
\end{tabular}




\begin{tabular}{|c|c|c|c|c|}
\hline & & & carcinoma & $\begin{array}{c}\text { diffuse tumors } \\
98.4 \% \\
\text { nodular tumors } \\
99.7 \% \\
\text { massive tumors } \\
98.7 \%\end{array}$ \\
\hline $\begin{array}{c}\text { Shapira } \\
2020\end{array}$ & & & $\begin{array}{c}\text { Healthy, cyst, } \\
\text { hypodense me- } \\
\text { tastasis }\end{array}$ & \\
\hline
\end{tabular}

\section{CONCLUSION}

In recent years, significant efforts have been made towards the development of the liver CAD system. Machine learning plays a vital role in liver $\mathrm{CAD}$ because organs may not be signified precisely by a simple equation or formulae. This study contributed an extensive literature review on the development of liver CAD systems from abdominal CT. The literature review reveals that performance of $\mathrm{CNN}$ in recent liver $\mathrm{CAD}$ system is good. Only some diseases like HCC, Cyst and hemangioma are considered by many authors. Diseases like cholangiocarcinoma, FNH, Fatty liver, cirrhosis and other diseases are not focused by many authors. Therefore, there is a requirement for a liver CAD system to characterize the benign tumor from the malignant tumor, which considers the rare diseases. In future deep learning will play a major role in liver CAD system. In this future work, we aim to find liver CAD system using deep learning model which characterize benign and malignant tumor from abdominal $\mathrm{CT}$ that consider rare liver diseases also.

\section{REFERENCES}

1. Alahmer, H., Ahmed, A.: Computer-Aided Classification of Liver Lesions from CT Images Based on Multiple ROI. Procedia Computer Science 90, 80 -86 (2016).

2. Al-Shabi, MA.: A Novel CAD System for Detection and Classification of Liver Cirrhosis using Support Vector Machine and Artificial Neural Network. IJCSNS International Journal of Computer Science and Network Security 19(8), 18-23 (2019).

3. Anwar, SM., Majid, M., Qayyum, A., Awais, M., Alnowami, M., Khan, MK.: Medical Image Analysis using Convolutional Neural Networks: A Review. Journal of medical systems 42(226), (2018).

4. Bray, F., Ferlay, J., Soerjomataram, I., Siegel, RL., Torre, LA., Jemal, A.: Global cancer statistics 2018: GLOBOCAN estimates of incidence and mortality worldwide for 36 cancers in 185 countries. CA Cancer J Clin 68, 394-424 (2018).

5. Brunetti, A., Carnimeo, L., Trotta, GF., Bevilacqua, V.: Computer-Assisted Frameworks for Classification of Liver, Breast and Blood Neoplasias via Neural Networks: a Survey based on Medical Images. Neurocomputing 335, 274-298 (2019).

6. Chang, CC., Chen, HH., Chang, YC., Yang, MY., Lo, CM., Ko, WC., Lee, YF., Liu, KL., Chang, RF.:
Computer-aided diagnosis of liver tumors on computed tomography images. Computer Methods and Programs in Biomedicine 145,45-51 (2017).

7. Chen, EL., Chung, PC., Chen, CL., Tsai, HM., Chang, CI.: An Automatic Diagnostic System for CT Liver Image Classification. IEEE Transactions on Biomedical Engineering, 45(6), 783-794 (1998).

8. Das, A., Acharya, UR., Panda, SS., Sabut, S.: Deep learning based liver cancer detection using watershed transform and Gaussian mixture model techniques. Cognitive Systems Research 54, 165175 (2019).

9. Dong, X., Zhou, Y., Wang, L., Peng, J., Lou, Y., Fan, Y.: Liver Cancer Detection using Hybridized Fully Convolutional Neural Network based on Deep learning framework. Special Section on Deep Learning Algorithms For Internet of Medical Things, IEEE Access Vol 8, 129889- 129898 (2020).

10. Fujita, H., Zhang, X., Kido, S., Hara, T., Zhou, X., Hatanaka, $Y$., Xu, R.: An Introduction and Survey of Computer-aided Detection/Diagnosis (CAD). In: International Conference on Future Computer, Control and Communication, 200-205 (2010).

11. Gletsos, M., Mougiakakou, SG., Matsopoulos, GK., Nikita, KS., Nikita, AS., Kelekis, D.: A Computer-Aided Diagnostic System to Characterize CT Focal Liver Lesions: Design and Optimization of a Neural Network Classifier. IEEE Transactions on Information Technology in Biomedicine 7(3), 153$162(2003)$

12. Gletsos, M., Mougiakakou, SG., Matsopoulos, GK., Nikita, KS., Nikita, AS., Kelekis, D.: Classification Of Hepatic Lesions From Ct Images Using Texture Features And Neural Networks. In: 2001 Proceedings of the 23rd Annual EMBS International Conference, Istanbul, Turkey, 2748-2751 (2001).

13. Gorunescu, F., Belciug, S., Gorunescu, M., Badea, $R$.: Intelligent decision-making for liver fibrosis stadialization based on tandem feature selection and evolutionary-driven neural network. Expert Systems with Applications 39:12824-12832 (2012).

14. Gunasundari, S., Suganya Ananthi, M.: Comparison and Evaluation of Methods for Liver Tumor Classification from CT Datasets. International Journal of Computer Applications 39(18), 46-51 (2012).

15. Gunasundari, S., Janakiraman, S.: A Study of Textural Analysis Methods for the Diagnosis of Liver Diseases from Abdominal Computed Tomography. 
International Journal of Computer Applications 74(11), 7-12 (2013).

16. Gunasundari, S., Janakiraman, S., Meenambal, S.: Velocity Bounded Boolean PSO for improved feature selection in liver and kidney disease diagnosis. Expert system with Application 56, 28-47 (2016).

17. Gunasundari, S., Janakiraman, S., Meenambal, S.: Multiswarm heterogeneous binary PSO using WinWin approach for improved feature selection in Liver and Kidney disease diagnosis. Computerized Medical Imaging and Graphics 70,135-154 (2018).

18. Gunasundari, S., Janakiraman, S., Meenambal, S.: Embedded Binary PSO Integrating classical methods for Multilevel Improved Feature Selection in liver disease diagnosis. Int. J. of Biomedical Engineering and Technology, 31(2) 105 - 136, (2019).

19. Huang, YL., Chen, JH., Shen, WC.: Diagnosis of Hepatic Tumors With Texture Analysis in Nonenhanced Computed Tomography Images. Academic Radiology 13(6), 713-720 (2006).

20. Kondo, T., Ueno, J., Takao, S.: Hybrid GMDHtype neural network using artificial intelligence and its application to medical image diagnosis of liver cancer. SI International, 1101-1106 (2011).

21. Kumar, SS., Moni, RS.: Diagnosis of Liver Tumor from CT Images Using Fast Discrete Curvelet Transform. IJCA Special Issue on Computer Aided Soft Computing Techniques for Imaging and Biomedical Applications CASCT, 2(4), 1173-1178 (2010).

22. Kumar, SS., Moni, RS., Rajeesh, J.: Liver Tumor Diagnosis by Gray Level and Contourlet Coefficients Texture Analysis. In: International Conference on Computing, Electronics and Electrical Technologies, 557-562 (2012).

23. Kumar, SS., Moni, RS., Rajeesh, J.: An automatic computer-aided diagnosis system for liver tumors on computed tomography images. Computers and Electrical Engineering, 39:1516-1526 (2013).

24. Kuo, WJ.: Computer-Aided Diagnosis for Feature Selection and Classification of Liver Tumors in Computed Tomography Images. In: Proceedings of IEEE International Conference on Applied System Innovation, 1207-1210 (2018).

25. Lee, CC., Chen, SH., Chia YC.: Classification of Liver Disease from CT Images Using a Support Vector Machine. Journal of Advanced Computational Intelligence and Intelligent Informatics 11(4), 396-402 (2007a).

26. Lee, CC., Chiang, YC., Tsai, CL., Chen, SH.: Distinction of Liver Disease from CT Images Using Kernel-based Classifiers. International Journal of Intelligent Computing in Medical Sciences \& Image Processing, 1(2), 113 - 120 (2007 b).

27. Lee, S., Choe, EK., Kim, SY., Kim, HS., Park, KJ., Kim D.: Liver imaging features by convolutional neural network to predict the metachronous liver metastasis in stage I-III colorectal cancer patients based on preoperative abdominal CT scan. In: The 18th Asia Pacific Bioinformatics Conference Seoul, Korea, 1-14 (2020).
28. Li, J., Sun, J., Shen, N., Chen, E., Zhang, Y.: A $C A D$ System for Liver Cancer Diagnosis Based on Multi-phase CT Images Features with BP Network. In: 11th International Conference on Intelligent Human-Machine Systems and Cybernetics (IHMSC), 67-70 (2019).

29. Li, J., Wu, Y., Shen, N., Zhang, J., Chen, E., Sun, J., Deng, Z., Zhang, Y.: A fully automatic computer-aided diagnosis system for hepatocellular carcinoma using convolutional neural networks. Biocybernetics and Biomedical Engineering 40(1), 238-248 (2020).

30. Mala, K., Sadasivam V.: Automatic Segmentation and Classification of Diffused Liver Diseases using Wavelet Based Texture Analysis and Neural Network. In: IEEE Indicon 2005 Conference, Chennai, India, 216-219 (2005).

31. Mala, K., Sadasivam, V., Alagappan, S.: Neural Network based Texture Analysis of Liver Tumor from Computed Tomography Images. World Academy of Science, Engineering and Technology 13, 767-774 (2008).

32. Mougiakakou, SG., Valavanis, I., Nikita, KS., Nikita, A., Kelekis, D.: Characterization of CT Liver Lesions Based on Texture Features and a Multiple Neural Network Classification Scheme. In: Proceedings of the 25' Annual International Conference of the IEEE EMBS Cancun, Mexico 12871290 (2003),

33. Mougiakakou, SG., Valavanis, IK., Nikita, A., Nikita, KS.: Differential diagnosis of CT focal liver lesions using texture features, feature selection and ensemble driven classifiers. Artificial Intelligence in Medicine 41, 25-37 (2007).

34. Nayantara, PV., Kamath, S., Rajagopal, KV.: Computer-Aided Diagnosis of liver lesions using CT images: A systematic review. Computers in Biology and Medicine 127 (2020).

35. Rajathi, GI., Jiji, GW.: Chronic Liver Disease Classification Using Hybrid Whale Optimization with Simulated Annealing and Ensemble Classifier. Symmetry 11(1), 1-21 (2019).

36. Rathore, S., Iftikhar, MA., Hussain, M., Jalil, A.: Texture analysis for liver segmentation and classification: a survey. Frontiers of Information Technology 121-126 (2011).

37. Saba, T.: Recent advancement in cancer detection using machine learning: Systematic survey of decades, comparisons and challenges. Journal of Infection and Public Health 13(9), 1274-1289 (2020).

38. Sadeque, ZA., Khan, TI., Hossain, QD., Turaba MY.: Automated Detection and Classification of Liver Cancer from CT Images using HOG-SVM model. In: 5th International Conference on Advances in Electrical Engineering (ICAEE), Dhaka, Bangladesh, 21-26 (2019).

39. Sayed, GI., Hassanien, AE., Schaefer, G.: An Automated Computer-Aided Diagnosis System for Abdominal CT Liver Images. In: International Conference on Medical Imaging Understanding and Analysis , Loughborough, UK, 68-73 (2016).

40. Shapira, N., Fokuhl, J., Schulthei, M., Stefanie, B., Kopp, FK., Pfeiffer, D., Dangelmaier, J., Pahn, G., 
Sauter, AP., Renger, B., Fingerle, AA., Rummeny, EJ., Albarqouni, S., Navab, N., Noël, PB.: Liver lesion localisation and classification with convolutional neural networks: a comparison between conventional and spectral computed tomography. Biomedical Physics \& Engineering Express 6(1), 1-10 (2020).

41. Stewart, B., Wild, CP.: World cancer report 2014.

42. Stoean, C., Stoean, R., Lupsor, M., Stefanescu, H., Badea, R.: Feature selection for a cooperative coevolutionary classifier in liver fibrosis diagnosis. Computers in Biology and Medicine 41, 238-246 (2011).

43. Subbiah Bharathi, V., Ganesan, L.: Orthogonal moments based texture analysis of CT liver images. Pattern Recognition Letters 29, 1868-1872 (2008).

44. Valavanis, I., Mougiakakou, SG., Nikita, KS., Nikita, A.: Computer Aided Diagnosis of CT Focal Liver Lesions by an Ensemble of Neural Network and Statistical Classifiers. In: IEEE International Joint Conference on Neural Networks (IEEE Cat. No.04CH37541), Budapest, 3, 1929-1934 (2004).

45. Wang, L., Zhang, Z., Liu, J., Jiang, B., Duan, X., Xie, Q., Hu, D., Li, Z.: Classification of Hepatic Tissues from CT Images Based on Texture Features and Multiclass Support Vector Machines. In: Proceedings of International Symposium on Neural Networks: Advances in Neural Network, (2009).

46. Yamashita, R., Mittendorf, A., Zhu, Z., Fowler, KJ., Santillan CS., Sirlin CB., Bashir MR., Do, KGR.: Deep convolutional neural network applied to the liver imaging reporting and data system (LI RADS) version 2014 category classification: a pilot study. Abdominal Radiology 45(1),24-35 (2020).

47. Zhou, X., Zhang, Y., Shid, M., Shi, H., Zheng, Z.: Early detection of liver disease using data visualisation and classification method. Biomedical Signal Processing and Control 11, 27-35 (2014). 